\title{
GESTIÓN TECNOLÓGICA PARA ACOMPASAR UN DESARROLLO INDUSTRIAL ACELERADO: EL RETO DEL NUEVO MILENIO PARA LOS PAÍSES EN VÍA DE DESARROLLO (Segunda Parte)
}

Fernando Machado*

* Director de Servicios Tecnológicos Investment and Technology Promotion Division, Unido - Viena, Junio de 1997

**Ingeniera Industrial de la Universidad Industrial de Santander, Msc. RPI N.Y.

Profesora de la Escuela de Ingeniería Industrial de la Universidad del Valle
El presente ensayo corresponde a las secciones 3 y 4 del documento de trabajo escrito por Fernando Machado, publicado en nuestro número anterior. Los derechos exclusivos de traducción y reproducción del documento fueron concedidos a esta publicación expresamente por el autor.

Traducido por Martha Cecilia Gómez de García ** 
EL PAPEL DE LAS INNOVACIONES ESTRATÉGICAS

La Figura 1 muestra una tipología de innovaciones tecnológicas basada en (1) el grado de capacidad en gestión tecnológica requerida para ejecutarlas exitosamente y (2) su impacto en la competitividad sostenible de las empresas.

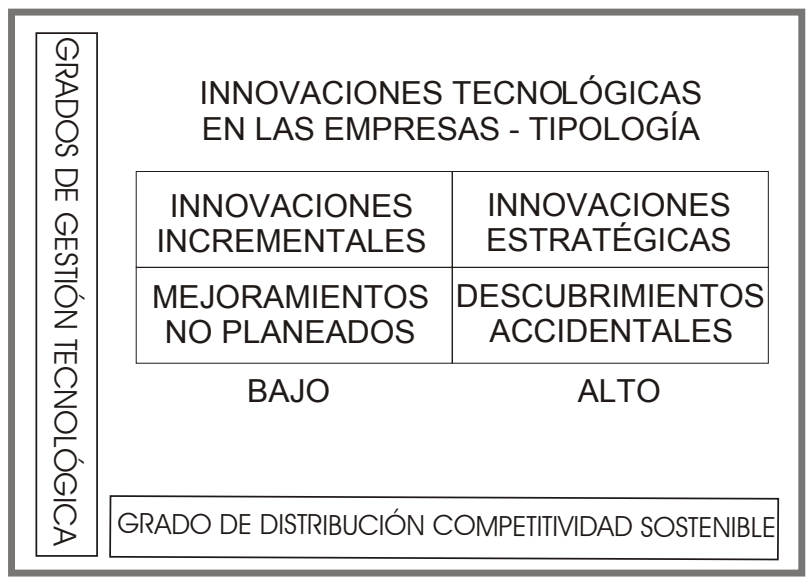

Figura 1

Los mejoramientos no planeados surgen de una variedad de fuentes - ideas en la ducha, creatividad de los trabajadores, quejas de los clientes, de la participación Ad- Honorem en asociaciones con otras organizaciones, etc. Requieren una capacidad limitada en gestión tecnológica y generalmente producen impacto no óptimo e insostenible en la competitividad de la empresa, bajo retorno en el uso de los activos de los socios y no incorporan soluciones ambientalmente durables.

Los descubrimientos accidentales corresponden a innovaciones no planeadas que producen un impacto representativo a corto plazo en las metas de la empresa descritas anteriormente. Estos resultan normalmente de una mezcla accidental de tecnologías y otros eventos fortuitos y también requieren de una capacidad limitada de gestión tecnológica

Las innovaciones incrementales están tan ampliamente aceptadas que han sido erróneamente adoptadas como la "receta" para la competitividad empresarial por la mayoría de empresarios de los países en vía de desarrollo. Por consiguiente lo que la mayoría de las organizaciones llama "innovación", realmente se limita a innovaciones incrementales.

Sin demeritar su importancia relativa para la obtención de ganancias de corto plazo en productividad, calidad y rentabilidad y la consecución de una rápida credibilidad en el proceso de cambio y la construcción de capacidad para administrar este cambio, las innovaciones incrementales en sí mismas, no contribuyen significativamente a la competitividad sostenible de la empresa, la responsabilidad ecológica y el retorno en el uso de los activos de los socios.

La razón es que estas innovaciones están dirigidas a mejorar la eficiencia operacional de la compañía, éstas se caracterizan por la aplicación de estándares de calidad para cumplir las especificaciones del producto y la producción establecida en los procesos de manufactura, por la optimización de estos procesos en cuanto a la minimización de desperdicio y energía, por la adopción de tecnologías limpias de fuentes externas, por el mejoramiento del diseño de los productos, por la actualización de los equipos y por la adopción de las mejores prácticas internacionales. Más aún, dichas innovaciones comprenden frecuentemente desarrollo "cosmético" de productos, el cual se manifiesta generalmente en nuevos empaques, formas, colores y sabores de los productos existentes. 
Las innovaciones incrementales involucran algo más que mejoramientos ligeros en el producto existente y normalmente tienen un impacto entre bajo y moderado sobre la productividad, la calidad y el crecimiento del Ingreso. Un modelo del nuevo año de cualquier automóvil existente es un ejemplo de una innovación incremental. Las compañías de alimentos introducen regularmente productos innovados incrementalmente y varios estudios indican que cerca del $90 \%$ de todos los "nuevos productos" son realmente extensiones de línea.

Para alcanzar estos mejoramientos, las empresas acuden a técnicas relacionadas con gestión total de la calidad ("kanban" y justo a tiempo), a sistemas de costeo por actividades, a ingeniería concurrente, auditorías energéticas, producción limpia, tecnologías de la información, "empoderamiento" del recurso humano (medido sobre la base de su conocimiento tácito), encuestas a los clientes y muchas otras.

Cuando se administran efectivamente, estas innovaciones incrementales redundan en ganancias de calidad y productividad, las cuales aumentan la rentabilidad y la satisfacción del cliente. Más aún, sientan las bases para la adopción de una cultura de cambio por parte de la empresa. La acumulación de innovaciones incrementales se utilizaba como "fórmula de éxito" en el pasado, cuando los mercados protegidos mantenían fuera los competidores internacionales y el crecimiento industrial así obtenido eran evolucionarias, no revolucionarias.

No obstante, no es extraño en estos tiempos que las empresas que realizan estas innovaciones incrementales exitosamente sean forzadas a cerrar o abandonar el negocio en un período corto después de agotar sus beneficios.

Este es normalmente el resultado de prestar poca atención a la evolución del contexto externo del negocio (incluyendo el contexto de negocios de los clientes propios), a las oportunidades y amenazas del negocio ocasionadas por discontinuidades tecnológicas y de mercado, a la posible reconstrucción de la empresa a la par con la cadena de comercialización, con el fin de reposicionarse a sí misma frente a los desarrollos de proveedores y clientes y a no ser capaz de traducir todo esto en innovaciones tecnológicas estratégicas, apalancadas en las habilidades medulares de la empresa.

Es ampliamente reconocido que las innovaciones incrementales generan un tipo de "miopía" para oportunidades de negocio que tiene como resultado el enfocarse demasiado en el bienestar de los productos de hoy y la amenaza de la competencia actual. Muy pocas compañías entienden cómo moverse más allá de esta "miopía" del negocio inducida por la innovación incremental y están por consiguiente restringidas permanentemente por esta "dictadura" del incrementalismo, incapaces de prestar atención a los cambios del contexto mencionados anteriormente.

Más aún, estas innovaciones se apoyan en la imitación de lo que las "mejores" compañías están haciendo para competir, pero como se describió anteriormente, la imitación tecnológica no puede proveer ni la diferenciación de mercado requerida por la competitividad, ni los descubrimientos requeridos por la nueva revolución industrial. Como consecuencia, la transferencia de tecnología por sí misma perdió significado. En estos tiempos, la búsqueda de "recetas tecnológicas" o soluciones tecnológicas "de mostrador" pueden capacitar a una empresa 
para producir cierto producto acorde con determinadas especificaciones y estándares, pero éstas no garantizan un centavo en las ventas, dejando de lado los beneficios de la nueva revolución industrial. La diferenciación de mercado y el desarrollo acelerado requieren entonces, no sólo transferencia de tecnología - incluidas tecnologías limpias - sino también investigación, desarrollo, la incorporación del conocimiento tácito de los trabajadores y su integración para añadir o crear valor para los clientes actuales y futuros más allá de los cambios en el producto, el proceso o equipo. En principio, cualquier fuente potencialmente significativa de diferenciación, estructura de costos, logística interna, canales de distribución, flexibilidad de entrega y otros- debería tenerse en cuenta para cambios tecnológicos estratégicos.

Por tanto, la contribución de las innovaciones incrementales al nivel competitivo de las empresas en el corto, mediano y largo plazo, a la comodidad ambiental sostenible, a la rentabilidad de sus sociedades y a la industrialización acelerada depende de su correlación con un plan estratégico que las combine apropiadamente con innovaciones (descubrimientos) accidentales.

\section{INNOVACIONES ESTRATÉGICAS}

Las innovaciones estratégicas son procesos en curso en los cuales una compañía sintetiza la observación de la industria y la tecnología con el conocimiento del mercado. Como un resultado, puede posicionarse para producir nuevos descubrimientos en tecnologías, productos y negocios requeridos por la nueva revolución industrial. Las compañías que aprovechan la fuerza de la innovación estratégica genera dramáticas ganancias, crecimiento a tasas que superan la competencia y son las primeras en reconocer y actuar con respecto a nuevas industrias emergentes a ser creadas por la nueva revolución industrial.

A diferencia de la creatividad genérica, las innovaciones estratégicas requieren inteligencia tecnológica y de mercados, toma de riesgos, apertura para mirar el mundo de una manera fresca y nueva, estrategias integradas, sistemas, características especiales en la cultura organizacional, recursos y la voluntad de actuar con convicción acerca de oportunidades de negocio futuras.

Las compañías capaces de innovar estratégicamente pueden entender su papel en la construcción del futuro. Mirando la evolución tanto de las industrias como de las tecnologías y los requerimientos de la nueva revolución industrial, se logra conocer posibles escenarios futuros y muchas empresas utilizan tendencias para diseñar nuevos portafolios de negocio los cuales están balanceados entre descubrimientos y oportunidades incrementales. Ellas reconocen tendencias complejas, analizan sus implicaciones y actúan focalizadas en alcanzar un estado futuro deseado que pueda proveer ventajas competitivas y sostenibilidad ambiental simultáneamente. Más aún, estas compañías refuerzan tendencias existentes, como base para definir y moldear tendencias futuras ventajosas. Más que responder a tendencias de la industria, dichas empresas participan en la nueva revolución industrial lo cual se requiere para salvar tanto el sistema económico global como el planeta.

La administración de innovaciones estratégicas requiere ir más allá del análisis de la estructura del negocio incluyendo tasas de crecimiento de la demanda y participación en el mercado, patrones de rivalidad, alianzas estratégicas, aspectos de propiedad intelectual, entrada, mantenimiento y barreras de salida, flujos de tecnología, el análisis de la rentabilidad relativa y otras ventajas de las 
diferentes posiciones en la cadena de valormás allá de estudios del cliente y gruposobjetivo, más allá del desarrollo del proveedor y cliente y aún más lejos de la restructuración del negocio mediante reposicionamiento en la cadena de valor.

Las compañías en el mundo, que realmente conocen su potencial de gestión de la innovación tecnológica, valoran la importancia de oportunidades futuras cuando evalúan la capacidad de sus organizaciones.

Ellas adoptan continuamente un portafolio equilibrado de oportunidades cercanas y de largo plazo, el cual fortalece y amplía sus habilidades medulares en nuevas áreas de negocio.

Para aprovechar el poder de la innovación estratégica, una compañía debe actuar en la visión (en el futuro) y en la introspección (en las necesidades de los clientes actuales y futuros). La combinación de ambas posiciones le permite conceptualizar un futuro deseado y crear los nuevos productos y negocios requeridos para materializarlo en una manera proactiva. Las miradas externa e interna han demostrado ser procesos fluídos, cambiantes, interrelacionados y complementarios.

La visión externa proviene de entender las tendencias en tecnología, demografía, regulación, estilos de vida y condiciones de contexto - tales como las descritas en relación con el futuro del sistema económico globalque pueden ser aprovechadas para reescribir las reglas de la industria y crear nuevos espacios competitivos. Como un proceso, ello involucra el examen continuo de las tendencias críticas y el desarrollo de un conjunto único de habilidades y tecnologías medulares que le permitan a la compañía materializar y conducir dichas tendencias.

Tanto en los países en vía de desarrollo como en los desarrollados, la asistencia del gobierno para facilitar este entendimiento a través de la formulación conjunta de políticas tecnológicas, incluyendo elementos de monitoreo y vigilancia tecnológica, ha demostrado ser un factor importante.

En particular, desarrollar una visión no es sencillo. No se trata de un proceso analítico de una sola vez que se obtiene mediante un simple estudio o programa de investigación. Es una amalgama continua de muchas perspectivas, una fusión colaborativa de hechos y proyecciones.

Por otra parte la introspección es un entendimiento profundo del porqué el comportamiento del cliente es como es, o porqué puede llegar a ser diferente en el futuro. En muchas compañías que quieren ser "orientadas al cliente", la retroalimentación bien intencionada del cliente en realidad restringe los esfuerzos de innovación estratégica. Cuando las organizaciones estudian su mercado, el 99\% de las veces el cliente puede unicamente articular su deseo de lo que en esencia es innovación incremental. No es de sorprenderse que los clientes rara vez pidan algo que represente un descubrimiento innovador.

De acuerdo con esto, las compañías comprometidas con encausar las oportunidades de una nueva revolución industrial mediante innovación estratégica deben constantemente profundizar su conocimiento de las necesidades y deseos que sus clientes no pueden ni articular. La opinión tradicional del cliente no es suficiente.

Por consiguiente, las innovaciones estratégicas requieren un análisis prospectivo de tecnologías, priorizado de acuerdo con su impacto en los factores competitivos existentes y futuros, la identificación de oportunidades y amenazas a los negocios 
existentes y nuevos, creados como resultado de discontinuidades de mercado y tecnología. Ello involucra la identificación y fortalecimiento de las competencias medulares de la empresa, el diagnóstico de la posición tecnológica competitiva de la compañía, la definición de las respectivas estrategias tecnológicas, portafolios de proyectos de riesgo controlado y roles de socios en innovación.

Estas actividades representan un esfuerzo complejo de riesgo significativo, que demanda información especializada, recursos humanos, materiales y financieros que no pueden proveerse por mecanismos de libre mercado. Esto es particularmente cierto para empresas de países en vía de desarrollo, la mayoría de las cuales de tamaño pequeño o mediano, donde el limitado nivel de educación de los empresarios condiciona su percepción acerca de (a) el rol que juegan diferentes tecnologías en su negocio, (b) la visión con respecto a futuros desarrollos de estas tecnologías, (c) la necesidad de buscar y tener acceso a métodos adecuados de priorización de necesidades tecnológicas, (d) la necesidad de una implementación profesionalmente efectiva de los proyectos tecnológicos de desarrollo acelerado y muchos otros aspectos cruciales que se requieren para definir e implementar con éxito innovaciones tecnológicas estratégicas .

La gerencia de estos procesos de innovación tecnológica estratégica, que involucra la mezcla de investigación y desarrollo con transferencia de tecnología en proporciones diversas, llevadas a cabo a través de esfuerzos multifuncionales, que normalmente traspasan varios límites organizacionales de empresas aliadas dentro del respectivo cluster (clientes, proveedores), es un proceso muy complejo y riesgoso. Dados los costos, el riesgo y los vacíos de información al interior de la firma para el aprendizaje y la innovación en mercados libres, todas las firmas, pero particularmente las PYMES, tenderán a invertir poco en estos procesos de innovación tecnológica requeridos para aprovechar las nuevas oportunidades de negocio creadas por la nueva revolución industrial.

Este requisito de "aprender-a-aprender" representa la falla más importante de los mecanismos de libre mercado en el acondicionamiento de un campo de acción llano para las empresas de países en vía de desarrollo.

De acuerdo con lo anterior, existe una amplia evidencia que la mayoría de PYMES en los países en vía de desarrollo no está involucrada en planeación más allá de un año. En sus agendas no hay proyectos de innovación y menos de la mitad llevan a cabo actividades permanentes de mejoramiento de la calidady la productividad a través de proyectos de innovación incremental.

Este vacío de aprendizaje e innovación sólo puede cerrase con apoyo externo, tal como se viene realizando en países como Estados Unidos, Canadá, Dinamarca, Francia, Suecia, Italia, Japón, Corea y malasia, entre otros.

\section{PREPARÁNDOSE PARA UN DESARROLLO ACELERADO}

En vista de lo anterior, el mayor dilema de los países en vía de desarrollo para sacar provecho de los beneficios de la nueva revolución industrial mediante un desarrollo acelerado, es la falta de capacidad de sus empresas para efectivamente llevar a cabo innovaciones tecnológicas estratégicas. Como se ha planteado, éstos son los principales actores involucrados y, al igual que en los países desarrollados, sus capacidades de innovación tecnológica deberían ser fomentadas por el estado. Así mismo, el punto de partida es inevitable es la creación de 
capacidades de gestión tecnológica estratégica (o gestión estratégica de la innovación tecnológica) al nivel de la empresa.

Una vez tomadas las decisiones sobre dirección, asociaciones, recursos e impacto esperado sobre los objetivos del negocio, lo que sigue es el compromiso de la empresa con la innovación tecnológica de gestión tecnológica, proveer los esquemas de financiación requeridos.

Ello implicará desde luego la búsqueda y utilización de todos los incentivos y servicios puestos a disposición de este proceso, a través de instrumentos específicos de política, programas de desarrollo de clientes e instituciones de apoyo a la industrialización. mismas empresas y estimular mecanismos para la creación de negocios de nuevas tecnologías, entre otras iniciativas.

La Figura 2 resume el ciclo virtual requerido para cambiar el actual esquema mental de concierne a la gestión de innovaciones tecnológicas estratégicas.

Se estima que la nueva revolución industrial multiplicará por diez la complejidad de la gestión tecnológica. Los requerimientos cuantitativos para los países en desarrollo, en términos de recurso humano capacitado son realmente astronómicos. Ellos tendrán que satisfacer las necesidades de las empresas existentes, de las nuevas empresas, de agentes del sistema de innovación tales como centros de I+D, empresas consultoras,
Consecuentemente, la política de innovación tecnológica de los países en desarrollo debería privilegiar el desarrollo de una cultura de innovación estratégica al interior de las empresas, apoyar el desarrollo de sus capacidades de gestión tecnológica, proveer los esquemas de financiación requeridos para para equilibrar los riesgos inherentes involucrados, estimular la conformación de posibles escenarios futuros y la visión de oportunidades de negocio relacionadas, respaldar pilotos y proyectos de gestión tecnológica estratégica para demostración, fomentar asociaciones entre empresas con miras a estimular transferencia y aprendizaje en gestión tecnológica, promover encuestas de gestión tecnológica, investigación y premios con plena participación de todas las
ESTRATEGIAS PARA ESTIMULAR GT - NIVEL PLANTA

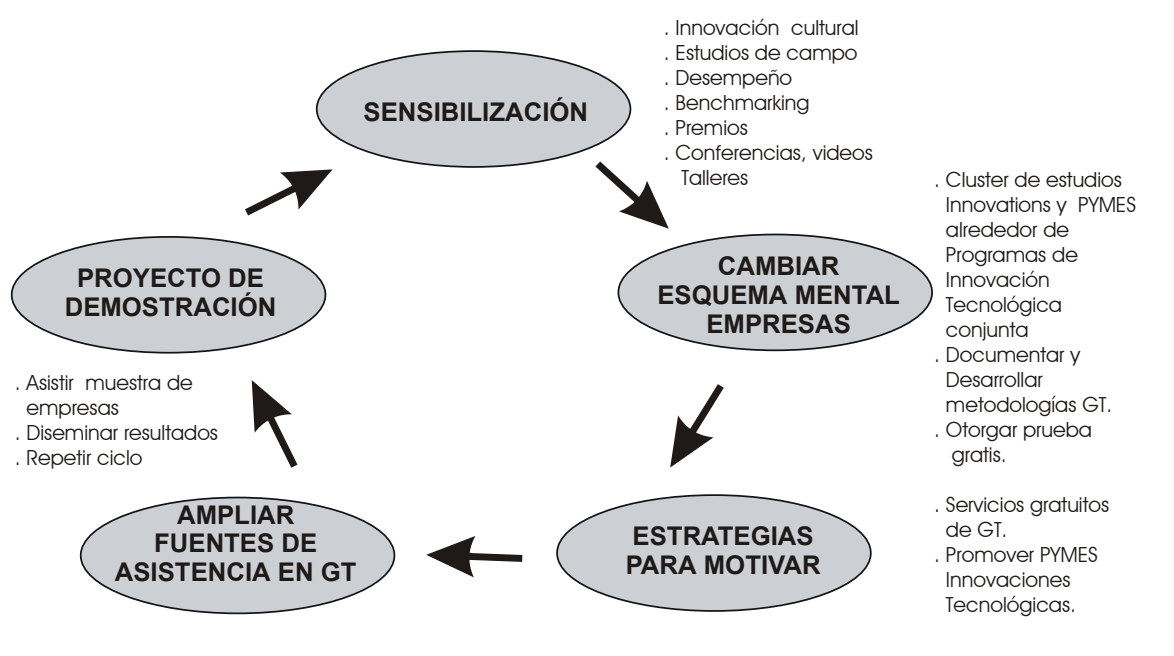

Figura 2

consejos de normalización y metrología, instituciones financieras, agentes de política científica y tecnológica, incubadoras y muchas otras.

A través de una apropiada definición de política, también tendrán que crearse y administrarse apropiadamente mediante el 
fortalecimiento de habilidades en gestión tecnológica, capacidades masivas de I+D, transferencia de tecnología, servicios tecnológicos tales como el establecimiento de nuevos estándares, certificación y pruebas, patentamiento, gestión de alianzas empresariales estratégicas, desarrollo de capacidades emprendedoras y financiación de capital de riesgo. En concordancia con lo cual, debe redefinirse el papel de las respectivas organizaciones regionales, nacionales e internacionales.

El riesgo de las inversiones mencionadas anteriormente es muy bajo. Aún en el evento improbable de que la nueva revolución industrial no se materialice y prevalezca la evolución de los patrones actuales que se describían en la Parte 11, la capacidad de administrar efectivamente la innovación tecnológica permanecerá siendo la capacidad medular para una competitividad sostenible y la supervivencia de las empresas alrededor del mundo.

1 Ver primera parte de este artículo en la Revista "Ingeniería y Competitividad", Volumen 2 - No. 1 - Mayo de 2000.

\section{BIBLIOGRAFÍA}

A.O. KRUEGER, American Enterprise Institute. "American Trade Policy: A Tragedy in the Making". 1998.

Business Week. "Forecasting Especially Dismal atDownturns". 30/09/96.

C. HANDY, The Economist Review. "The Invisible Fist". 15/02/97.

D. JOHNSTON, IHT. "From the Marshall Plan to True Globalization". 26/03/97.

F. LEWIS, IHT. "Tell Us About Nations Getting Ahead Together". 17/03/96.
F. MACHADO, UNIDO Internal Document. "Concepts for Thematic Priority" 4 Innovation, Productivity and Quality for International Competitiveness". 13/01/97.

F. MACHADO SELA. "La Política Industrial y la Política Tecnólogica: Los Retos Actuales". Capítulos Industria, Tecnología y Competitividad. October December 1995.

Financial Times. "Over-Capacity Stalks tehe Economics of Asian Tigers". 17/06/97.

Financial Times Editorial. "Passing the Global Toroh". 30/1 1/96.

G. HAMEL, HBR. "Strategy as Revolution". July August 1996.

G. HAMEL and C.K. PRAHALAD, HBR Press. "Competing for the Future". 1994.

H.E. DALY, BEACON. "Beyond Growth: The Economics of Sustainable Development". 1996.

IHT. "Winking atAnti-Helms Law?". 27/02/97.

J. CAUVANAGH, IHT. "Globalization: Fine for Some and Bad for Many". 24/01/97.

J. HOAGLAND, IHT. "The World's Bottom Line: Money is Everything". 12-13/04/97.

J. KYNGE, Financial Times. "Nervous Malaysians SettoRide the Storm". 21-22/06/97.

J. SARKIS, Technovation. "Manufacturing Strategy and Environmental Consciousness". Vol. 15, No. 2, 1995.

L.C. THUROW, MORROW. "The Future of Capitalism How Today's Economic Forces Shape Tomorrow's World". 1996.

M. FRANCE BAUD, The UNESCO Courier. "Market 
M. FRANCE BAUD, The UNESCO Courier. "Market Globalization". 11/96.M.

PORTER, HBR. "What is Strategy?". Nov.Dec. 1996.

M. RENNER, NORTON. "Fighting for Survival". 1996.

P.D. SUTHERLAND, IHT. "Beyond the Market, A DifferentKind of Equity". 20/02/97.

R.B. SHAPIRO, CEO, MONSANTO, HBR. "Growth through Global Sustainability". Jan Feb. 1997.

R. DAGE, IHT. "America's Bullying Trade Tactics". $11 / 06 / 98$.

R. KUTTNER, KNOPF, "Everything for Sale". 1996.

S.L. HART, HBR. "Beyond Greening: Strategies for a Sustainable World". Jan. Feb. 1997.

The Economist. "Dodgy Regimes, Dodgy Tactics" The United States' new sanctions on Cuba, Iran and Libya are dangerous. 01/06/96.

The Economist. "The Backlash in Latin America". $30 / 11 / 96$.

V. FORRESTER, UNESP (Librairie, Artheme Fayard). "O Horror Economico". 1996.

"WTO Report on World Trade, 1996".

W. CHANKIM, R. MAUBORGNE, HBR. "Value Innovation: The Strategic Logic of High Growth". Jan. Feb. 1997.

W. GREIDER, SIMON and SCHUSTER. "One World, Ready or Not The Manic Logic of Global Capitalism". 1997.

W. GREIDER, IHT. "What is Globalization Fatal Flaw? Oversupply". 25/02/97.

W. PFAFF, IHT. "The Tide Turns Against Irresponsible Worship of the Market". 